\title{
Calcium is the switch in the moonlighting dual function of the ligand-activated receptor kinase phytosulfokine receptor 1
}

\author{
Victor Muleya' ${ }^{1}$, Janet I Wheeler ${ }^{1}$, Oziniel Ruzvidzo², Lubna Freihat ${ }^{1}$, David T Manallack' ${ }^{1}$ Chris Gehring ${ }^{3}$ \\ and Helen R Irving ${ }^{1 *}$
}

\begin{abstract}
Background: A number of receptor kinases contain guanylate cyclase (GC) catalytic centres encapsulated in the cytosolic kinase domain. A prototypical example is the phytosulfokine receptor 1 (PSKR1) that is involved in regulating growth responses in plants. PSKR1 contains both kinase and GC activities however the underlying mechanisms regulating the dual functions have remained elusive.

Findings: Here, we confirm the dual activity of the cytoplasmic domain of the PSKR1 receptor. We show that mutations within the guanylate cyclase centre modulate the GC activity while not affecting the kinase catalytic activity. Using physiologically relevant $\mathrm{Ca}^{2+}$ levels, we demonstrate that its GC activity is enhanced over two-fold by $\mathrm{Ca}^{2+}$ in a concentration-dependent manner. Conversely, increasing $\mathrm{Ca}^{2+}$ levels inhibits kinase activity up to 500-fold at $100 \mathrm{nM} \mathrm{Ca}^{2+}$.

Conclusions: Changes in calcium at physiological levels can regulate the kinase and GC activities of PSKR1. We therefore propose a functional model of how calcium acts as a bimodal switch between kinase and GC activity in PSKR1 that could be relevant to other members of this novel class of ligand-activated receptor kinases.
\end{abstract}

Keywords: Calcium, Guanylate cyclase, Kinase, PSKR1

\section{Findings}

In higher and lower eukaryotes, many receptor kinases contain a putative guanylate cyclase catalytic centre encapsulated in the C-terminal part of the kinase domain (Figure 1A). Candidate receptor kinases with this novel type of overlapping dual-domain architecture are not uncommon since Arabidopsis thaliana alone is estimated to have more than 40 members of this new class of proteins [1]. Membrane-bound members of this class of proteins have a typical architecture containing an extracellular ligand binding domain, a single transmembrane spanning domain and an intracellular catalytic kinase domain [2].

In plants, four members of the guanylate cyclaseembedded receptor kinases have been shown to possess

\footnotetext{
*Correspondence: helen.irving@monash.edu

'Monash Institute of Pharmaceutical Sciences, Monash University, 381 Royal Parade, Parkville, VIC 3052, Australia

Full list of author information is available at the end of the article
}

a low level of intrinsic guanylate cyclase activity in vitro; these are the brassinosteroid receptor (BRI1; BRASSINOSTEROID INSENSITIVE 1) [3], the wall associated kinase-like10 (WAKL10) [4], the elicitor peptide 1 receptor (PepR1) [5] and the phytosulfokine receptor 1 (PSKR1) [6]. All of these molecules have a primary function as kinases and predominantly fold as kinase molecules $[1,7]$. In another recent study, no guanylate cyclase activity was detected in the BRI1 kinase domain where the assay conditions used favoured kinase activity and the construct lacked the cytoplasmic domains necessary to promote dimerization [7]. Dimerization and/or activation of a molecular switch to turn down the kinase activity may be necessary to generate conformational folding required for guanylate cyclase activity [1].

PSKR1 recognizes the secreted cell proliferation agent, phytosulfokine (PSK), containing sulphated tyrosine residues [8,9] and is essential for cell growth [10-12]. Brassinosteroid signalling enhances PSK expression and 
A

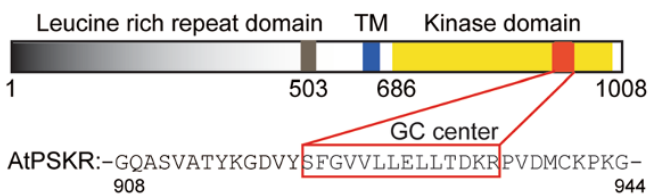

C

E

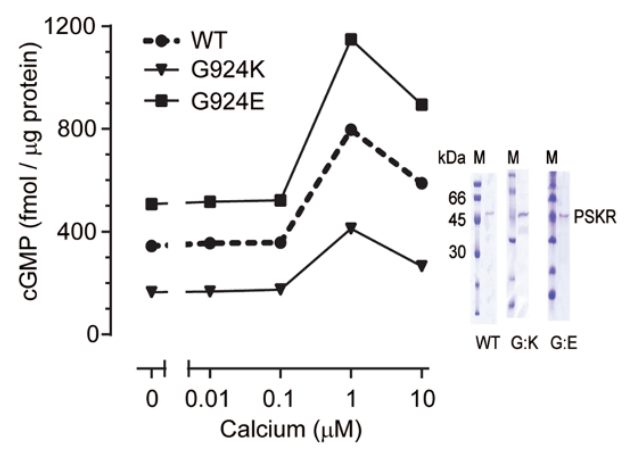

B

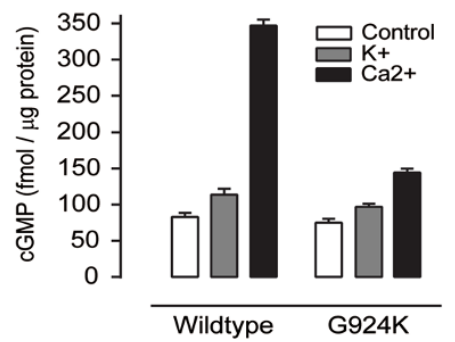

D

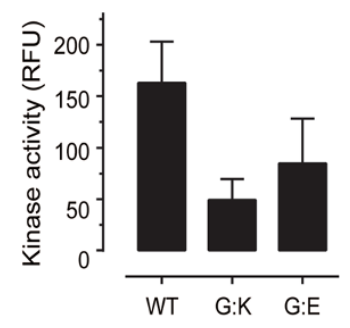

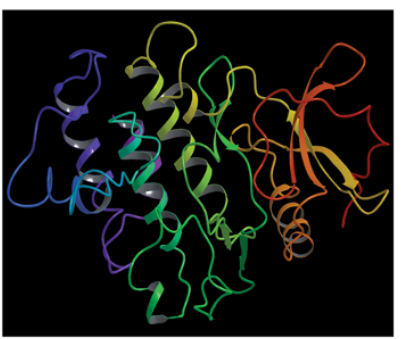

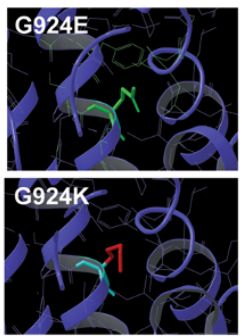

Figure 1 Effect of calcium on guanylate cyclase activity of PSKR1. A Schematic diagram of PSKR1 featuring the sequence motif of the guanylate cyclase catalytic centre and the immediately surrounding amino acids (908-944). TM refers to the transmembrane domain and the ligand binding domain occurs in the extracellular region from residues 503 to 517. B Effect of cations on guanylate cyclase activity of PSKR1. The cytoplasmic domain of PSKR1 (residues 683 to 1008) was expressed as either wild-type His-tagged SUMO-fused protein or the mutant protein (G924K) prepared as previously described [6]. Calcium significantly enhanced guanylate cyclase activity and the G924K mutant had significantly less activity than the wild-type (mean \pm s.e.m., $n=3 ; P<0.0001$ two-way ANOVA, Sidak's multiple comparison test). C The effect of calcium on the guanylate cyclase activity of wild-type and mutant (G924K and G924E) PSKR1 (residues 686 to 1008) was measured at increasing calcium concentrations buffered with EGTA and $\mathrm{Mg}^{2+}$. The curves and all the treatments at 1 and $10 \mu \mathrm{M}$ are significantly different (mean \pm s.e.m. (error bars within symbol), $\mathrm{n}=3$ independent experiments; $P<0.0001$ two-way ANOVA, Tukey-Kramer multiple comparison test). The purified wild-type and mutant PSKR1 (residues 686-1008) molecules $(1 \mu \mathrm{g}$ ) were analysed by SDS-PAGE. D The kinase activity of the G924K or G924E mutants (residues 686-1008) determined at $8 \mathrm{nM}$ free calcium were not significantly different to the wild-type (mean \pm s.e.m., $\mathrm{n}=3$ independent experiments; $P=0.1532$ one-way ANOVA). E Homology model of the cytoplasmic domain of PSKR1 was developed based on tomato resistance protein Pto. The homology model was mutated in the guanylate cyclase domain to show the effect of G924E (tolerated) and G924K (steric hindrance). The red colour associated with the lysine residue indicates steric hindrance and strain.

PSKR1 dependent quiescent centre cell division [13] and PSK is involved in attenuating stress responses [14] with roles in both immune and developmental processes $[15,16]$. PSKR1 mediated signalling elicits increases in guanosine $3^{\prime}, 5^{\prime}$-cyclic phosphate (cGMP) in isolated mesophyll protoplasts and transfection of protoplasts with full length PSKR1 results in raised endogenous levels of cGMP [6]. Recently, the kinase activity of PSKR1 has been shown to be essential for PSK signalling in vivo [17]. However, the underlying mechanisms regulating the overlapping dual functions of guanylate cyclase-embedded receptor kinases have remained elusive. Here we use PSKR1 as a representative member of this novel class of receptor kinases to unravel the biochemical conditions that enable the dual functions. We show that calcium has opposing effects on the kinase and guanylate cyclase activities of PSKR1. We propose a functional model of how calcium acts as a bimodal molecular switch between these two activities so that they do not occur concurrently. 


\section{Calcium enhances GC activity}

When examining the regulation of the cytoplasmic domain of PSKR1 in vitro, it was previously found that the kinase activity was inhibited by cGMP [6], suggesting the potential for bimodal modification of the dual activities. Further support for this notion was obtained when the effect of ionic conditions on the cytoplasmic domain of recombinant AtPSKR1 [TAIR:AT2G02220; GenBank: NP_1783300.1] was examined, as guanylate cyclase activity was enhanced in the presence of $5 \mathrm{mM} \mathrm{Ca}^{2+}$ but not $\mathrm{K}^{+}$ions (Figure 1B and see Additional file 1: Supplementary Methods). This finding was suggestive that the guanylate cyclase activity of the protein is modulated by a calcium-specific rather than an ion-specific effect. To test the hypothesis that calcium ions modify the enzymatic activities of PSKR1, we used calcium buffer systems to precisely control free calcium ion levels and measured the activities of the cytoplasmic domain of AtPSKR1 (see Additional file 1: Supplementary Methods). Free calcium concentrations were determined using the Maxchelator program taking into account the temperature, $\mathrm{pH}$ and ionic strength of the calcium buffer [18]. Guanylate cyclase activity occurred in the absence of calcium and was considerably enhanced at 1 and $10 \mu \mathrm{M} \mathrm{Ca}^{2+}$ but not at lower concentrations in the wild-type protein (Figure 1C). However, the in vitro guanylate cyclase activity of PSKR1 is still rather low when compared to canonical membrane bound guanylate cyclases. The $\mathrm{G}$ residue in the catalytic motif of GCs is predicted to determine substrate specificity for GTP $[19,20]$ and it was previously shown that when the $G$ residue (924) in the catalytic motif was mutated to $K$ (G924K), it had decreased guanylate cyclase activity [6]. In this study, the G924K mutation conferred reduced guanylate cyclase activity at all calcium concentrations tested however the pattern of response was the same as wild-type protein (Figure 1B and C). Since the positively charged substitution decreased guanylate cyclase activity, we tested the effect of a negatively charged substitution with the expectation that guanylate cyclase activity may be retained. This was indeed the case as the G924E mutant showed enhanced guanylate cyclase activity with an overall similar pattern of response to all physiological calcium concentrations (Figure 1C). Thus increasing free calcium from 100 $\mathrm{nM}$ to $1 \mu \mathrm{M}$ enhanced guanylate cyclase activity by approximately two-fold. Both mutations had no effect on kinase activity when tested at $8 \mathrm{nM} \mathrm{Ca}^{2+}$ (one way ANOVA, $P=0.1532$; Figure 1D) being a calcium concentration favouring kinase activity (see Figure $2 \mathrm{~A}$ ); suggestive that the main function of this region in the kinase domain is to generate cGMP.

We developed a homology model of the kinase domain of PSKR1 (Figure 1E) based on its $41.2 \%$ identity to the crystal structure of tomato resistance protein Pto (for Pseudomonas syringae pv tomato) kinase [PDB: 3HGK]
[21] (see Additional file 1: Supplementary Methods). When a G924K mutation was incorporated into the model, the molecule became strained due to the steric hindrance encountered by the $\mathrm{K}$ residue whereas a negatively charged residue at this position (G924E) was tolerated (Figure 1E) and providing structural reasons for the measured catalytic activities of PSKR1 (Figure 1B and C).

\section{Calcium suppresses kinase activity}

We then determined the effect of precisely controlled calcium levels on the kinase activity of wild-type PSKR1 molecule. The kinase activity of the wild-type protein was completely suppressed at and above $100 \mathrm{nM}$ free calcium (Figure 2A). Kinase activity was rapidly suppressed in response to increased calcium ion concentrations as the same preparation was measured in "zero" calcium where kinase activity was present before being subjected to $10 \mu \mathrm{M} \mathrm{Ca}^{2+}$, resulting in inhibition of the activity. Addition of EDTA to reduce the calcium ion levels resulted in a relatively small increase in kinase activity which did not return to original levels (Figure 2B).

In conclusion, our findings indicate that the kinase activity of PSKR1 is directly inhibited by increases in free calcium ions and importantly, the guanylate cyclase activity is enhanced at similar calcium concentrations, indicative of a reciprocal regulation of the dual functionality of this molecule. The free calcium concentration in the cytoplasm of plant cells is estimated to be in the magnitude of 50 to $100 \mathrm{nM}$ and to increase approximately two to five-fold upon stimulation, depending on particular signature profiles [22]. In plants, calcium regulates a number of protein kinases either directly or indirectly as a means of modulating their biological activity during signal transduction [22-25]. The kinase domain of PSKR1 also contains a calmodulin binding domain that has been shown to interact with all isoforms of calmodulin and is essential for normal growth [17]. Calmodulin is activated by calcium but how calmodulin binding or calcium (this study) directly modulates kinase activity of PSKR1 is currently unknown. Changes in calcium have direct and opposing effects on the alternate intrinsic activities of PSKR1 with the kinase activity being completely suppressed at the concentrations stimulating guanylate cyclase activity. Hence changes in calcium ions act as a direct molecular switch that enables activation of alternate downstream responses.

An important part of any signalling network is the ability to turn down the cascade at appropriate junctures. In bi-functional molecules such as PSKR1, the fact that different intracellular conditions favour one function over the other may allow these molecules to turn on/off their alternate signalling cascades in response to the cellular environment and thereby fine-tune their cognate signalling networks. Furthermore, the initial 

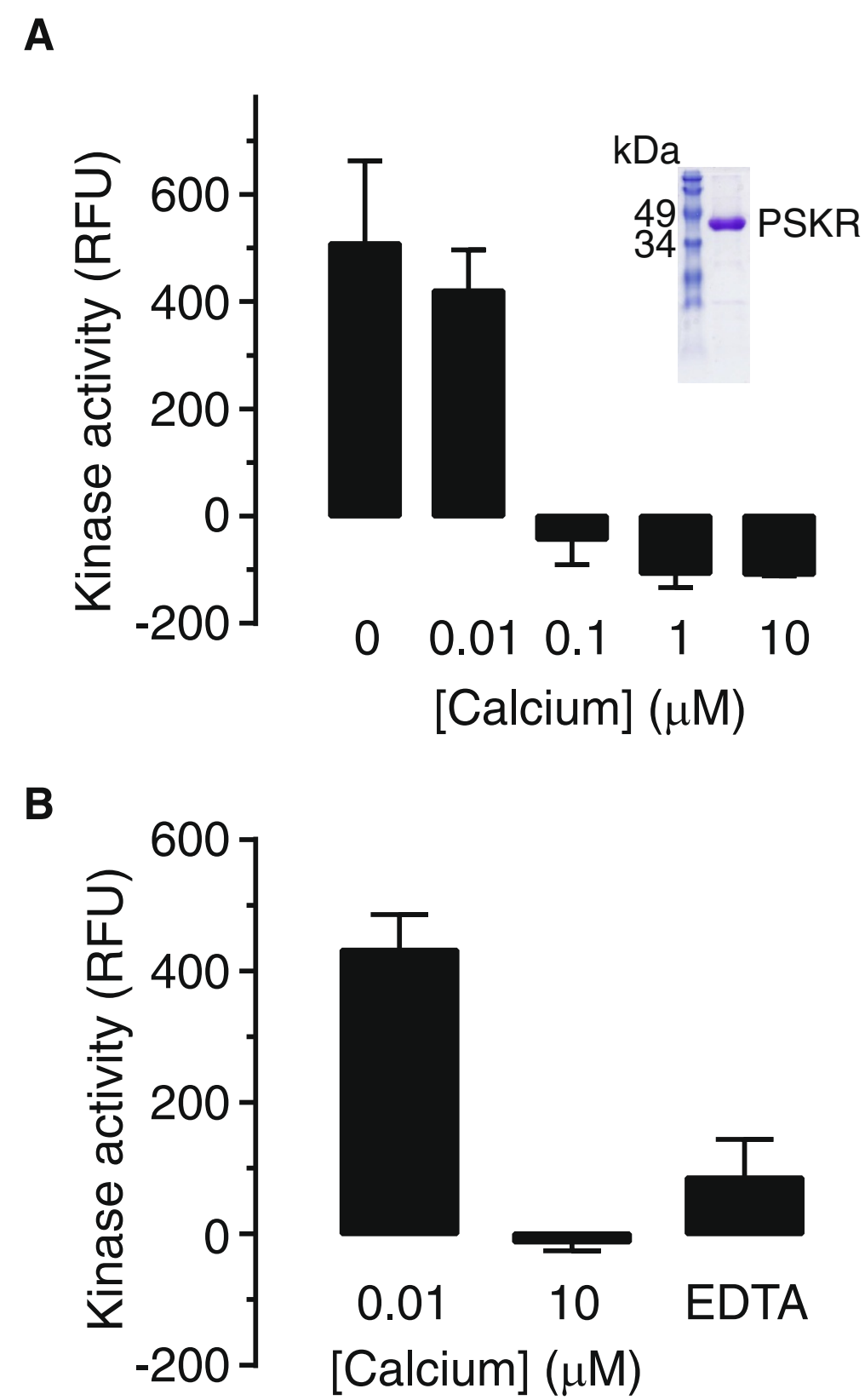

Figure 2 Effect of calcium on kinase activity of PSKR1. A Kinase activity (relative fluorescence units (RFU)) of the cytoplasmic domain of wild-type PSKR1 (residues 686-1008) was measured at increasing calcium concentrations buffered with EGTA and $\mathrm{Mg}^{2+}$. Kinase activity was significantly reduced at calcium concentrations greater than $0.01 \mu \mathrm{M}$ (mean \pm s.e.m., $\mathrm{n}=3$ independent experiments; $P=0.0008$, one-way ANOVA, Tukey-Kramer multiple comparison test). The inset shows the SDS-PAGE analysis of the purified cytoplasmic domain of PSKR1 $(5 \mu \mathrm{g})$. B Suppression of wild-type PSKR1 kinase activity by calcium. Kinase activity of wild-type PSKR1 (residues 686-1008) was determined when incubated in zero calcium and then subjected to $10 \mu \mathrm{M}$ free calcium before $10 \mathrm{mM}$ EDTA was added. Kinase activity was significantly reduced by the calcium treatment $($ mean \pm s.e.m., $\mathrm{n}=3 ; P=0.0013$ one-way ANOVA, Tukey-Kramer multiple comparison test).

finding that cGMP, a product of guanylate cyclase activity, inhibits the kinase activity of PSKR1 [6], provides another switch that augments the effect of calcium and thus enabling PSKR1 to shuttle between its alternate signalling networks. The physiological relevance of such switches is supported by recent observations that PSKR1 mediates a switch from growth and development to plant defence responses $[15,16]$. The fact that the activation of plant defence responses is dependent on changes in both cytosolic calcium and cGMP [26] further substantiates the importance of the PSKR1 switch mediated by changes in intracellular calcium. 


\section{Additional file}

Additional file 1: Supplementary methods.

\section{Abbreviations}

BRI1: BRASSINOSTEROID INSENSITIVE 1 (brassinosteroid receptor); cGMP: Guanosine 3',5'-cyclic phosphate; GC: Guanylate cyclase; PSK: Phytosulfokine; PSKR1: Phytosulfokine receptor 1 .

\section{Competing interests}

The authors declare that they have no competing interests.

\section{Authors' contributions}

CG and HRI conceived the study and prepared the manuscript with VM; VM, JIW, OR and LF undertook the experiments; DTM developed the homology model; all authors were involved in data analysis, reading and revision of the manuscript. All authors read and approved the final manuscript.

\section{Acknowledgements}

This work was supported by the Australian Research Council's Discovery funding scheme [project numbers DP0878194, DP110104164] and the National Research Foundation South Africa [grant numbers CSUR78843; IRF2009021800047]. The authors also acknowledge helpful discussions with MDW Griffin, I Jennings, L Kwezi, E Leung, C Marondedze, Y-F Mok, L Thomas and $Y H$ Wang, and technical support from $\mathrm{K}$ Govender.

\section{Author details}

${ }^{1}$ Monash Institute of Pharmaceutical Sciences, Monash University, 381 Royal Parade, Parkville, VIC 3052, Australia. ${ }^{2}$ Department of Biological Sciences, North-West University, Private Bag X2046, Mmabatho 2735, South Africa. ${ }^{3}$ Division of Biological and Environmental Sciences and Engineering, 4700 King Abdullah University of Science and Technology, 23955-6900 Thuwal, Kingdom of Saudi Arabia.

Received: 4 June 2014 Accepted: 11 September 2014

Published online: 23 September 2014

\section{References}

1. Wong A, Gehring C: The Arabidopsis thaliana proteome harbors undiscovered multi-domain molecules with functional guanylyl cyclase catalytic centers. Cell Commun Sig 2013, 11:48.

2. Gou X, He K, Yang H, Yuan T, Lin H, Clouse SD, Li J: Genome-wide cloning and sequence analysis of leucine-rich repeat receptor-like protein kinase genes in Arabidopsis thaliana. BMC Genomics 2010, 11:19.

3. Kwezi L, Meier S, Mungur L, Ruzvidzo O, Inving H, Gehring C: The Arabidopsis thaliana brassinosteroid receptor (AtBRI1) contains a domain that functions as a guanylyl cyclase in vitro. PLOS One 2007, 2:e449.

4. Meier S, Ruzvidzo O, Morse M, Donaldson L, Kwezi L, Gehring C: The Arabidopsis Wall Associated Kinase-Like 10 gene encodes a functional guanylyl cyclase and is co-expressed with pathogen defense related genes. PLoS One 2010, 5(1):e8904.

5. Qi Z, Verma R, Gehring C, Yamaguchi Y, Zhao Y, Ryan CA, Berkowitz GA: Ca ${ }^{2+}$ signaling by plant Arabidopsis thaliana Pep peptides depends on AtPepR1, a receptor with guanylyl cyclase activity, and cGMP-activated $\mathrm{Ca}^{2+}$ channels. Proc Nat Acad Sci USA 2010, 107:21193-21198.

6. Kwezi L, Ruzvidzo O, Wheeler Jl, Govender K, lacuone S, Thompson PE, Gehring C, Irving HR: The phytosulfokine (PSK) receptor is capable of guanylate cyclase activity and enabling cyclic GMP-dependant signaling in plants. J Biol Chem 2011, 286:22580-22588.

7. Bojar D, Martinez J, Santiago J, Rybin V, Bayliss R, Hothorn M: Crystal structures of the phosphorylated BRI1 kinase domain and implications for brassinosteroid signal initiation. Plant J 2014, 78:31-43.

8. Matsubayashi Y, Ogawa M, Morita A, Sakagami Y: An LRR receptor kinase involved in perception of a peptide plant hormone, phytosulfokine. Science 2002, 296:1470-1472.

9. Shinohara H, Ogawa M, Sakagami Y, Matsubayashi Y: Identification of ligand binding site of pytosulfokine receptor by on-column photoaffinity labeling. J Biol Chem 2007, 282:124-131.
10. Kutschmar A, Rzewuski G, Stührwohldt N, Beemster GTS, Inzé D, Sauter M: PSK-a promotes root growth in Arabidopsis. New Phytol 2009, 181:820-831.

11. Matsubayashi Y, Ogawa M, Kihara H, Niwa M, Sakagami Y: Disruption and overexpression of Arabidopsis phytosulokine receptor gene affects cellular longevity and potential for growth. Plant Physiol 2006, 142:45-53.

12. Hartmann J, Stührwohldt N, Dahlke RI, Sauter M: Phytosulokine control of growth occurs in the epidermis, is likely to be non-cell autonomous and is dependent on brassinosteroids. Plant J 2013, 73:579-590.

13. Heyman J, Cools T, Vandenbussche F, Heyndrickx KS, Van Leene J, Vercauteren I, Vandeauwera S, Vandepoele K, De Jaeger G, Van Der Straeten D, De Veylder L: ERF115 controls root quiescent center cell division and stem cell replenishment. Science 2013, 342:860-863.

14. Motose H, Iwamoto K, Endo S, Demura T, Sakagami Y, Matsubayashi Y, Moore KL, Fukuda $\mathrm{H}$ : Involvement of phytosulfokine in the attenuation of stress response during the transdifferentiation of Zinnia mesophyll cells into tracheary elements. Plant Physiol 2009, 150:437-447.

15. Igarashi D, Tsuda K, Katagiri F: The peptide growth factor, phytosulfokine, attenuates pattern-triggered immunity. Plant J 2012, 71:194-204.

16. Mosher S, Seybold H, Rodriguez P, Stahl M, Davies KA, Dayaratne S, Morillo SA, Wierzba M, Favery B, Keller B, Tax FA, Kemmerling B: The tyrosine-sulfated peptide receptors PSKR1 and PSY1R modify the immunity of Arabidopsis to biotrophic and necrotrophic pathogens in an antagonistic manner. Plant J 2013, 73:469-482.

17. Hartmann J, Fischer C, Dietrich P, Sauter M: Kinase activity and calmodulin binding are essential for growth signaling by the phytosulfokine receptor PSKR1. Plant J 2014, 78:192-202.

18. Bers DM, Patton CW, Nuccitelli R: A practical guide to the preparation of $\mathrm{Ca}^{2+}$ buffers. Methods Cell Biol 2010, 99:1-26.

19. Sunahara RK, Beuve A, Tesmer JJG, Sprang SR, Garbers DL, Gilman AG: Exchange of substrate and inhibitor specification between adenylyl and guanylyl cyclases. J Biol Chem 1998, 273:16332-16338.

20. Tucker $\mathrm{CL}$, Hurley JH, Miller TR, Hurley JB: Two amino acid substitutions convert a guanylyl cyclase, RetGC-1, into an adenylyl cyclase. Proc Nat Acad Sci USA 1998, 95:5993-5997.

21. Dong J, Xiao F, Fan F, Gu L, Cang H, Martin GB, Chai J: Crystal structure of the complex between Pseudomonas effector AvrPtoB and the tomato Pto kinase reveals both a shared and a unique interface compared with AvrPto-Pto. Plant Cell 2009, 21:1846-1859.

22. Kudla J, Batistic O, Hashimoto K: Calcium signals: the lead currency of plant information processing. Plant Cell 2010, 22:541-563.

23. Batistic O, Kudla J: Analysis of calcium signaling pathways in plants. Biochim Biophys Acta 1820, 2012:1283-1293.

24. Defalco TA, Bender KW, Snedden WA: Breaking the code: $\mathrm{Ca}^{2+}$ sensors in plant signalling. Biochem J 2010, 425:27-40.

25. Liese A, Romeis T: Biochemical regulation of in vivo function of plant calcium-dependent protein kinases (CDPK). Biochim Biophys Acta 1833, 2013:1582-1589.

26. Tena G, Boudsocq M, Sheen J: Protein kinase signaling networks in plant innate immunity. Curr Opin Plant Biol 2011, 14:519-529.

\section{doi:10.1186/s12964-014-0060-z}

Cite this article as: Muleya et al:: Calcium is the switch in the moonlighting dual function of the ligand-activated receptor kinase phytosulfokine receptor 1. Cell Communication and Signaling 2014 12:60.

\section{Submit your next manuscript to BioMed Central and take full advantage of:}

- Convenient online submission

- Thorough peer review

- No space constraints or color figure charges

- Immediate publication on acceptance

- Inclusion in PubMed, CAS, Scopus and Google Scholar

- Research which is freely available for redistribution 